\title{
BMJ Open Documenting and characterising gestational weight gain beliefs and experiences among Marshallese pregnant women in Arkansas: a protocol for a longitudinal mixed-methods study
}

\author{
Britni L Ayers, ${ }^{1}$ Cari A Bogulski, ${ }^{2}$ Lauren Haggard-Duff, ${ }^{3}$ Aline Andres, ${ }^{4}$ \\ Elisabet Børsheim (D) , ${ }^{4}$ Pearl A McElfish
}

To cite: Ayers BL, Bogulski CA, Haggard-Duff L, et al. Documenting and characterising gestational weight gain beliefs and experiences among Marshallese pregnant women in Arkansas: a protocol for a longitudinal mixedmethods study. BMJ Open 2020;10:e037219. doi:10.1136/ bmjopen-2020-037219

- Prepublication history for this paper is available online To view these files, please visit the journal online (http://dx.doi org/10.1136/bmjopen-2020037219).

Received 23 January 2020 Revised 15 June 2020 Accepted 14 August 2020
Check for updates

(C) Author(s) (or their employer(s)) 2020. Re-use permitted under CC BY-NC. No commercial re-use. See rights and permissions. Published by BMJ.

For numbered affiliations see end of article.

Correspondence to Dr Pearl A McElfish; pamcelfish@uams.edu

\section{ABSTRACT}

Introduction Arkansas has the largest population of Marshallese Pacific Islanders residing in the continental USA. The Marshallese have higher rates of obesity, type 2 diabetes, pre-term births, low birthweight babies, infant mortality, and inadequate or no prenatal care. Despite the high rates of cardiometabolic and maternal and child health disparities among Marshallese, there are no studies documenting gestational weight gain or perceptions about gestational weight gain among the Marshallese population residing in the USA.

Methods and analysis This paper describes the protocol of a mixed-methods concurrent triangulation longitudinal study designed to understand gestational weight gain in Marshallese women. The mixed-methods design collects qualitative and quantitative data during simultaneous data collection events, at both first and third trimester, and then augments that data with postpartum data abstraction. Quantitative and qualitative data will be analysed separately and then synthesised during the interpretation phase.

Ethics and dissemination The study used a community engaged approach approved by the University of Arkansas for Medical Sciences Institutional Review Board (\#228023). The research team will disseminate results to study participants, research stakeholders (clinics, faith-based organisations and community-based organisation), the broader Marshallese community and fellow researchers. Results will be disseminated to study participants through a one-page summary that show the aggregated research results using plain language and infographics.

\section{INTRODUCTION}

Arkansas has the largest population of Marshallese Pacific Islanders residing in the continental USA. ${ }^{2}$ Marshallese have several chronic health disparities, including higher rates of obesity, diabetes and hypertension. ${ }^{34}$ The Marshallese community is also disproportionately burdened by poor maternal health outcomes in comparison with other racial and ethnic groups. Specifically, the Marshallese

\section{Strengths and limitations of this study}

- This will be the first study to document gestational weight gain and perceptions about gestational weight gain among the Marshallese population residing in the USA.

- To address the health disparities experienced by the Marshallese community, the authors are using a community-based participatory research approach.

- This mixed-methods concurrent triangulation longitudinal design will allow the researchers to overcome the inherent weaknesses of using a singular, cross-sectional, qualitative or quantitative methodology.

- This study will be used to culturally tailor interventions to help Marshallese women achieve recommended gestational weight gain and reduce maternal and infant health disparities in Marshallese communities.

- The results of this study may or may not be generalisable to other Pacific Islander communities residing outside Arkansas.

have higher rates of pre-term births, low birthweight babies, infant mortality, and inadequate or no prenatal care. ${ }^{56}$

Excessive gestational weight gain (GWG) increases medical complications for the mother, ${ }^{7-9}$ and is associated with impaired glucose intolerance, ${ }^{10-12}$ delivery complications, increased healthcare costs, ${ }^{13}$ greater postpartum weight retention, ${ }^{14} 15$ higher incidence of obesity later in life ${ }^{16-19}$ and metabolic disease risk later in life. ${ }^{20}$ There is a strong association between excessive GWG and gestational diabetes mellitus (GDM). ${ }^{1021}$ The prevalence of GDM among Pacific Islander communities has not been well documented in the current literature. The limited studies conducted have documented that Pacific Islanders residing in Samoa, Republic of the 
Marshall Islands (RMI), Hawaii, Washington and California have higher rates of GDM compared with other racial/ethnic demographics. These studies have documented rates of GDM as high as $13.7 \%$ in Pacific Islanders compared with $5.8 \%$ for the general population in the continental USA. ${ }^{22-30}$

Excessive GWG increases obesity and negative health outcomes in the offspring. ${ }^{31}$ Children born to mothers with obesity are more likely to develop obesity later in life and suffer metabolic impairment. ${ }^{32}$ Children born to mothers with excessive GWG are at higher risks of rapid weight gain, obesity and hypertension later in life..$^{33-37}$

There is limited literature documenting GWG in Pacific Islanders. Hawley et al found that $78 \%$ of Samoan women (of whom $86 \%$ were overweight or obese) exceeded GWG guidelines. This study documented that greater GWG was associated with increased odds of caesarean section delivery and overweight/obese infants by age 12 months. ${ }^{38}$

Despite the high rates of obesity, diabetes and hypertension, ${ }^{3}$ and the high rates of maternal and child health disparities among Marshallese, there are no studies documenting GWG or perceptions about GWG among the Marshallese population residing in the USA.

Health disparities among the Marshallese are rooted in a complex history between the USA and the Marshallese. The USA military conducted nuclear testing in the Marshall Islands between 1946 and 1958, detonating 67 fission and thermonuclear devices equivalent to 7200 Hiroshima-sized bombs. ${ }^{39}$ As a result, areas of the Marshall Islands were contaminated, disrupting their dominant food sources of fish and locally grown plants. ${ }^{40}$ The Marshallese diet and lifestyle in the RMI shifted to a Western diet high in fat and simple carbohydrates, and a more sedentary lifestyle after the nuclear testing. ${ }^{41}$ Some studies document higher rates of miscarriage, pre-term birth and birth defects immediately after the nuclear contamination. ${ }^{39}$ In addition, GWG research is constrained by Marshallese distrust of the healthcare system and scientific research. ${ }^{42}$

To overcome these barriers and address the health disparities experienced by the Marshallese community, the authors will use a community-based participatory research (CBPR) approach. CBPR is a research approach seeking to involve community partners in all aspects of the research process. ${ }^{44}$ This type of research is uniquely suited for engaging indigenous and/or immigrant populations to overcome historical trauma. As part of the CBPR collaborative, the research team has spent the past 5 years meeting with Marshallese community members to determine and prioritise the community's primary health concerns. Maternal health was identified as a top priority.

\section{METHODS AND ANALYSIS}

This study has been approved by the University of Arkansas for Medical Sciences Institutional Review Board (\#228023).
Patient and public involvement

No patient involvement.

\section{Study aims}

The aims of this study are to document and characterise GWG among Marshallese in Arkansas via ten domains: (1) pregnancy experience; (2) diet during pregnancy; (3) physical activity during pregnancy; (4) perceptions of appropriate GWG; (5) information sources about GWG; (6) weight control behaviour; (7) tools and resources for GWG management; (8) doctor-patient communication; (9) GWG goal setting and (10) basic demographics.

\section{Approach}

A CBPR approach will be used in the design and implementation of this study. The study will be guided by the Healthy Start Community Action Network (CAN) that includes both local healthcare professionals as well as Marshallese community members; and an interprofessional research team that includes quantitative and qualitative researchers, as well as Marshallese community health workers (CHWs) to provide accurate translation of study materials and input on how to modify the study materials and protocol to be culturally appropriate for Marshallese participants. ${ }^{45}$

\section{Study design}

The study will use a mixed-methods concurrent triangulation longitudinal design. ${ }^{46}$ This design collects both qualitative and quantitative data during data collection events, at both first (6-12 weeks' gestation) and third trimester (30-36 weeks' gestation), and then augments that data with postpartum data abstraction. All participants will participate in both data collection events. Quantitative and qualitative data will be analysed separately and then synthesised during the interpretation phase. This mixedmethods concurrent triangulation longitudinal design will allow the researchers to overcome the inherent weaknesses of using a singular qualitative or quantitative methodology. ${ }^{47} 48$ Further, as knowledge and behavioural components can change over time during the course of the pregnancy, we will be able to capture these changes by using a longitudinal design via multiple time points of data collection.

\section{Data collection}

Data collection is anticipated to begin October 2020. Quantitative and qualitative (see the Instruments section) data will be collected at first and third trimester during the same data collection event by a female bilingual CHW. Height and weight measurements will be taken during first and third trimester data collection events. Participants will also be provided the option to sign a Health Insurance Portability and Accountability Act (HIPAA) release to access their maternal and neonatal medical records which will be obtained to abstract clinical information after the mother gives birth at 6 weeks post partum. 


\section{Instruments}

The quantitative surveys, qualitative interview guides, and abstraction and biometric templates were developed with intensive input from Marshallese stakeholders. After the instruments were initially drafted with stakeholders, the CBPR team met monthly with three female bilingual CHWs who will be implementing the data collection in-language with the participants. The CHWs reviewed the questions and discussed revisions. Both the first and third trimester surveys went through four revisions. The first trimester interview guide went through three revisions and the third trimester interview guide went through two revisions.

\section{Quantitative surveys}

The quantitative survey was adapted from The Glowing Study to be appropriate for Marshallese participants. ${ }^{49}$ The surveys will be implemented using Research Electronic Data Capture. ${ }^{50}$ Each survey will take approximately $30 \mathrm{~min}$ to complete. Surveys are divided into four domains that will measure: (1) basic demographics; (2) perceptions of appropriate GWG; (3) GWG goal setting and (4) weight control behaviours.

\section{Qualitative interview guides}

Interview guides are divided into eight domains: (1) pregnancy experience; (2) diet during pregnancy; (3) physical activity during pregnancy; (4) perceptions of appropriate GWG; (5) information sources used to make decision on GWG; (6) weight control behaviour and (7) tools and resources for GWG management. The third trimester has one additional domain: (8) doctor-patient communication.

\section{Biometric and abstracted data}

Height and weight will be measured during first and third trimester data collection events. The height and weight taken at first trimester will be used as a proxy for prepregnancy weight and will be used in conjunction with the height and weight taken and third trimester to calculate GWG. In addition, the research team will abstract medical record information about the mother and infant. The data abstracted from the mother will include: (1) date of first prenatal care; (2) number of prenatal care visits; (3) fasting glucose; (4) blood pressure; (5) gestational weeks at delivery; (6) complications; (7) GDM test results; (8) mothers' pre-maternal weight status; (9) the amount of weight gained (both cumulatively and per trimester); (10) timing of weight gained and (11) infant feeding intentions. For infants, data abstraction will include: (1) weight and height of infant and (2) birth/ medical complications.

Once data collection instruments were confirmed, CHWs translated them into the Marshallese language. After the data documents were translated, the CBPR team met with the female bilingual CHWs and conducted mock data collection events monthly over the course of 3 months. These mock data collection events served as training for the CHWs and allowed the team to evaluate any challenges in cultural nuance, comprehension and translations.

\section{Recruitment, consent and retention}

All study documents used for recruitment, consent and retention were developed in collaboration with Marshallese stakeholders (CAN and CHWs) using a CBPR approach. Participants will be recruited by female bilingual CHWs with extensive research training and trust within the Marshallese community. Fifty women will be recruited by the female bilingual CHWs, who will recruit at local clinics, faith-based organisations and communitybased organisations. The target of 50 was chosen because it will allow us to reach saturation across a diverse group of Marshallese women in Arkansas. ${ }^{38}$ Data saturation refers to the quality and quantity of information. Saturation occurs when redundancy is reached in data analysis and signals to researchers that data collection may cease. $^{52}$ Our previous work with Marshallese female participants has used 50 as a target enrolment number and this has demonstrated effective in reaching saturation. ${ }^{435153}$ Further, our team works closely with a biostatistician who has verified this is an appropriate target enrolment number for exploratory analyses. If saturation is not achieved among these 50 women, the CBPR team will recruit additional participants. The inclusion criteria are: (1) women who self-report as Marshallese; (2) 18 years of age or older and (3) pregnant. Exclusion criteria are: (1) conception with the use of fertility treatments; (2) multiple gestations and (3) use of medications known to influence fetal growth (eg, glucocorticoids, insulin, thyroid, hormones). Exclusion criteria were chosen as these components would qualify the participants as potential high-risk pregnancies.

Potential participants who meet the inclusion criteria will be offered the opportunity to join the study and complete the consent process. Trained female bilingual CHWs will provide each participant a copy of the consent for medical records in either/both English and Marshallese. The consent forms will use plain language. The female bilingual CHWs will read the consent aloud to the participants in the participant's language of choice (English or Marshallese).

The CBPR team will use an engaged approach to collaboratively develop a retention plan with Marshallese stakeholders. The retention plan specifies that all female bilingual CHWs responsible for recruitment and retention will be bilingual (Marshallese/English). Female bilingual CHWs will obtain each participant's contact information and preferred method of contact. Female bilingual CHWs will also collect contact information for at least two relatives and ask participants for permission to contact their relatives if needed. Confidentiality rules will be followed, and no participant information will be provided to relatives. Before each data collection visit, female bilingual CHWs will contact study participants about the upcoming data collection visit. If a participant 
withdraws, the study team will document who withdrew and why they withdrew. Marshallese participants will receive a $\$ 40$ gift card at each data collection event. The CBPR team has previously demonstrated the ability to recruit and retain more than 400 participants in a 3-year study with four data collection events over 14 months and maintain a retention rate of over $90 \% .^{54}$

\section{Data analysis}

\section{Abstraction and biometric}

Maternal and neonatal data for all participants will be abstracted at 6 weeks post partum. Gestational weight gain will be derived using weight at delivery minus earliest prenatal care visit weight and categorised as below, within or above the Institute of Medicine guidelines. Maternal and infant outcomes will be compared among women across the GWG categories in adjusted and unadjusted analyses with ORs and 95\% CI reported. Covariates will include parity, prior caesarean birth and pregestational diabetes.

\section{Data quality control}

Once all data have been analysed, a data quality control meeting will take place with all female bilingual CHWs to validate the interpretation of the qualitative and quantitative findings of the study.

\section{Quantitative}

While the primary purpose of collecting quantitative data will be to support and triangulate the qualitative data, exploratory analyses of the quantitative data will be conducted to contextualise the role of the perceptions, attitudes and behaviours of GWG in Marshallese pregnant women. Quantitative data analysis of the survey results will use descriptive as well as inferential statistical techniques including analysis of variance, $X^{2}$ tests, correlations, and logistic or linear regressions. The descriptive analyses will use frequencies and proportions to summarise the four domains: (1) basic demographics, including age, marital status, household size, education, employment status and place of birth; (2) perceptions of appropriate GWG; (3) GWG goal setting and (4) weight control behaviours. Inferential analyses will focus on assessing the relationship between Marshallese mothers' perceptions and behaviours toward GWG, including weight gain expectations, child feeding, perceptions of body size, locus of control in weight management, healthy eating, physical activity, and family support of diet and exercise practices. Inferential analyses will also examine mothers' perceptions and behaviours and associations with actual GWG and other health outcomes, such as blood pressure, complications at delivery, GDM test results and infant birth/medical complications. Demographic information will be considered for inclusion in any regression analyses conducted to adjust for the effects of age, socioeconomic status, and place of birth on perceptions, attitudes and behaviours toward GWG. Time-varying covariates and changes in those measures assessed during the first trimester and third trimester data collection events will be considered as additional predictors in inferential models. Additionally, changes in perceptions and behaviours related to GWG throughout pregnancy will be documented. These analyses will use a standard alpha level of 0.05, two-tailed, and will report effect sizes where informative. For longitudinal analyses, only respondents who participated in both data collection events will be included. For all analyses, the number of respondents who provided valid responses will be presented.

\section{Qualitative}

Qualitative data from interviews will be audio recorded and transcribed verbatim in the language it was spoken by a female bilingual CHW. Then any information transcribed in Marshallese will be translated into English. Three researchers with qualitative interview experience will start with initial coding, which consists of naming each data segment with short summations. This process helps organise the data for focused codes. The focused codes that emerge will be used to identify and develop the most salient categories within the data. ${ }^{556}$ The research team will collaboratively discuss the themes in order to ensure scientific rigour and intercoder agreement. There will be two primary coders and one confirmation coder. Then, using standard qualitative analysis, an inductive process will be used to identify and code emerging themes. The qualitative analytic approach will integrate inductive and deductive techniques, and the codebook will include a priori thematic codes that represent themes from the interview guide and emergent codes that capture unanticipated categories of analysis.

\section{Analysis plan}

The analysis for this longitudinal study will be twofold: (1) a cross-sectional analysis will take place analysing the data collected at first and third trimester and (2) a comparative analysis will be implemented to compare and contrast the data collected from both data collection events.

\section{STRENGTHS AND LIMITATIONS}

This will be the first study to document GWG and perceptions about GWG among the Marshallese population residing in the USA. This study will be used to culturally tailor interventions to help Marshallese women achieve recommended GWG and reduce maternal and infant health disparities in Marshallese communities. This mixed-methods concurrent triangulation longitudinal design will allow the researchers to overcome the inherent weaknesses of using a singular, cross-sectional, qualitative or quantitative methodology. The results of this study may or may not be generalisable to other Pacific Islander communities residing outside Arkansas. However, establishing evidence-based interventions designed for Pacific Islanders may also inform work with other disenfranchised and indigenous populations who have strong 
collectivist cultures, thus increasing the generalisability of the proposed research. ${ }^{57-61}$

\section{DISSEMINATION}

Effective dissemination is crucial to achieving research impact, and is a key component to conducting CBPR. The research team will use the Agency for Healthcare Research and Quality's Dissemination Planning Tool as the framework for our dissemination. ${ }^{62}$ Specifically, we will disseminate results to study participants, research stakeholders (clinics, faith-based organisations and community-based organisation), the broader Marshallese community and fellow researchers. Results will be disseminated to study participants through a one-page summary that shows the aggregated research results using plain language and infographics. To extend the reach, this information will be reviewed in a town hall meeting and disseminated using social media. No individual participant information will be shared and all confidentiality procedures will be maintained. The data will be published in peer-reviewed journal articles and presented at academic conferences.

\section{SUMMARY}

Marshallese are disproportionately burdened by poor maternal and infant health outcomes. Excessive GWG is a key modifiable risk factor for maternal and infant health disparities. However, GWG in USA Marshallese communities has not been well understood. This study will be used to culturally tailor interventions to help Marshallese women achieve recommended GWG and reduce maternal and infant health disparities in Marshallese communities.

\section{Author affiliations}

${ }^{1}$ College of Medicine, University of Arkansas for Medical Sciences Northwest, Fayetteville, Arkansas, USA

${ }^{2}$ Office of Community Health and Research, University of Arkansas for Medical Sciences Northwest, Fayetteville, Arkansas, USA

${ }^{3}$ College of Nursing, University of Arkansas for Medical Sciences Northwest, Fayetteville, Arkansas, USA

${ }^{4}$ Department of Pediatrics, University of Arkansas for Medical Sciences, Little Rock, Arkansas, USA

\section{Twitter Elisabet Børsheim @elborshe}

Acknowledgements We would like to acknowledge Lynda Riklon, Morda Netwon and Mell Jacklick for translations and future data collection.

Contributors BLA, AA and PAM conceptualised the design, acquisition of data and analysis, and formulated the research questions of the study. PAM and BLA wrote the manuscript. CAB, LH-D, AA and EB edited the manuscript. CAB, BLA and PAM will interpret and analyse the data once collected.

Funding The CBPR partnership support was provided from the University of Arkansas for Medical Sciences Translational Research Institute (grant UL1TR000039), which was funded through the NIH National Center for Research Resources and National Center for Advancing Translational Sciences. Research reported in this publication was also partially supported by the National Institute of General Medical Sciences of the National Institutes of Health under Award Number P20GM109096. The content is solely the responsibility of the authors and does not necessarily represent the official views of the National Institutes of Health.

Competing interests None declared.
Patient and public involvement Patients and/or the public were not involved in the design, or conduct, or reporting, or dissemination plans of this research.

Patient consent for publication Not required.

Provenance and peer review Not commissioned; externally peer reviewed.

Open access This is an open access article distributed in accordance with the Creative Commons Attribution Non Commercial (CC BY-NC 4.0) license, which permits others to distribute, remix, adapt, build upon this work non-commercially, and license their derivative works on different terms, provided the original work is properly cited, appropriate credit is given, any changes made indicated, and the use is non-commercial. See: http://creativecommons.org/licenses/by-nc/4.0/.

ORCID iDs

Elisabet Børsheim http://orcid.org/0000-0002-7842-0625

Pearl A McElfish http://orcid.org/0000-0002-4033-6241

\section{REFERENCES}

1 Craft D. The Marshallese population continues to grow in northwest Arkansas. 30. Northwest Asian Weekly, 2011.

2 Shulte B. For Pacific Islanders, hopes and troubles in Arkansas. The New York times, 2012. Available: https://www.nytimes.com/2012/07/ 05/us/for-marshall-islanders-hopes-and-troubles-in-arkansas.html

3 McElfish PA, Rowland B, Long CR, et al. Diabetes and hypertension in Marshallese adults: results from faith-based health screenings. $J$ Racial Ethn Health Disparities 2017;4:1042-50.

4 McElfish PA, Moore R, Laelan M, et al. Using CBPR to address health disparities with the Marshallese community in Arkansas. Ann Hum Biol 2018;45:264-71.

5 Nembhard WN, Ayers BL, Collins RT, et al. Adverse pregnancy and neonatal outcomes among Marshallese women living in the United States. Matern Child Health J 2019;23:1525-35.

6 Nembhard WN, McElfish PA, Ayers B, et al. Nuclear radiation and prevalence of structural birth defects among infants born to women from the Marshall islands. Birth Defects Res 2019;111:1192-204.

7 Flegal KM, Carroll MD, Kit BK, et al. Prevalence of obesity and trends in the distribution of body mass index among US adults, 1999-2010. JAMA 2012;307:491-7.

8 Basu S, Haghiac M, Surace P, et al. Pregravid obesity associates with increased maternal endotoxemia and metabolic inflammation. Obesity 2011;19:476-82.

9 Challier JC, Basu S, Bintein T, et al. Obesity in pregnancy stimulates macrophage accumulation and inflammation in the placenta. Placenta 2008;29:274-81.

10 Hedderson MM, Gunderson EP, Ferrara A. Gestational weight gain and risk of gestational diabetes mellitus. Obstet Gynecol 2010;115:597-604

11 Herring SJ, Oken E, Rifas-Shiman SL, et al. Weight gain in pregnancy and risk of maternal hyperglycemia. Am J Obstet Gynecol 2009;201:61.e1-61.e7.

12 Tovar A, Must A, Bermudez Ol, et al. The impact of gestational weight gain and diet on abnormal glucose tolerance during pregnancy in Hispanic women. Matern Child Health J 2009;13:520-30.

13 Chu SY, Bachman DJ, Callaghan WM, et al. Association between obesity during pregnancy and increased use of health care. $N$ Engl $J$ Med 2008;358:1444-53.

14 Gould Rothberg BE, Magriples U, Kershaw TS, et al. Gestational weight gain and subsequent postpartum weight loss among young, low-income, ethnic minority women. Am J Obstet Gynecol 2011;204:52.e1-52.e11.

15 Gunderson EP, Abrams B, Selvin S. The relative importance of gestational gain and maternal characteristics associated with the risk of becoming overweight after pregnancy. Int J Obes Relat Metab Disord 2000;24:1660-8.

16 Davis EM, Zyzanski SJ, Olson CM, et al. Racial, ethnic, and socioeconomic differences in the incidence of obesity related to childbirth. Am J Public Health 2009;99:294-9.

17 Gunderson EP, Abrams B. Epidemiology of gestational weight gain and body weight changes after pregnancy. Epidemiol Rev 2000;22:261-74.

18 Olson CM. Achieving a healthy weight gain during pregnancy. Annu Rev Nutr 2008;28:411-23.

19 Siega-Riz AM, Viswanathan M, Moos M-K, et al. A systematic review of outcomes of maternal weight gain according to the Institute of medicine recommendations: birthweight, fetal growth, and postpartum weight retention. Am J Obstet Gynecol 2009;201:339. e1-339.e14. 
20 Gunderson EP, Jacobs DR, Chiang V, et al. Childbearing is associated with higher incidence of the metabolic syndrome among women of reproductive age controlling for measurements before pregnancy: the cardia study. Am J Obstet Gynecol 2009;201:177. e1-177.e9.

21 Hedderson MM, Williams MA, Holt VL, et al. Body mass index and weight gain prior to pregnancy and risk of gestational diabetes mellitus. Am J Obstet Gynecol 2008;198:409.e1-409.e7.

22 Chang AL, Soon R, Kaneshiro B. The prevalence of gestational diabetes among Micronesians in Honolulu. Hawaii Med $J$ 2010;69:4-6.

23 Chang AL, Hurwitz E, Miyamura J, et al. Maternal risk factors and perinatal outcomes among Pacific Islander groups in Hawaii: a retrospective cohort study using statewide hospital data. $B M C$ Pregnancy Childbirth 2015;15:239.

24 Silva JK, Kaholokula Joseph Keawe'aimoku, Ratner R, et al. Ethnic differences in perinatal outcome of gestational diabetes mellitus. Diabetes Care 2006;29:2058-63.

25 Rao AK, Daniels K, El-Sayed YY, et al. Perinatal outcomes among Asian American and Pacific Islander women. Am J Obstet Gynecol 2006;195:834-8.

26 Tsitas M, Schmid BC, Oehler MK, et al. Macrosomic and low birth weight neonates in Pacific Islanders from Samoa: a case-control study. Arch Gynecol Obstet 2015;292:1261-6.

27 Wartko PD, Wong EY, Enquobahrie DA. Maternal birthplace is associated with low birth weight within racial/ethnic groups. Matern Child Health J 2017;21:1358-66.

28 Prevention CfDCa. National diabetes fact sheet: national estimates and general information on diabetes and prediabetes in the United States. Atlanta, GA: US Department of Health and Human Services, Center for Disease Control and Prevention, 2011.

29 UNICEF. Republic of the Marshall Islands: A Situational Analysis of Children, Youth \& Women, 2003. Available: https://evaw-globaldatabase.unwomen.org/en/countries/oceania/marshall-islands/2003/ a-situation-analysis-of-children-youth-and-women

30 Lavery JA, Friedman AM, Keyes KM, et al. Gestational diabetes in the United States: temporal changes in prevalence rates between 1979 and 2010. BJOG 2017;124:S92.

31 Catalano PM. Obesity and pregnancy--the propagation of a viscous cycle? J Clin Endocrinol Metab 2003;88:3505-6.

32 Catalano PM, Shankar K. Obesity and pregnancy: mechanisms of short term and long term adverse consequences for mother and child. BMJ 2017;356:j1.

33 Oken E, Taveras EM, Kleinman KP, et al. Gestational weight gain and child adiposity at age 3 years. Am J Obstet Gynecol 2007;196:322. e1-322.e8.

34 Mamun AA, O'Callaghan M, Callaway L, et al. Associations of gestational weight gain with offspring body mass index and blood pressure at 21 years of age: evidence from a birth cohort study. Circulation 2009;119:1720-7.

35 Oken E, Rifas-Shiman SL, Field AE, et al. Maternal gestational weight gain and offspring weight in adolescence. Obstet Gynecol 2008;112:999-1006.

36 Hull HR, Thornton JC, Ji Y, et al. Higher infant body fat with excessive gestational weight gain in overweight women. Am J Obstet Gynecol 2011;205:211.e1-211.e7.

37 Mamun AA, Mannan M, Doi SA. Gestational weight gain in relation to offspring obesity over the life course: a systematic review and biasadjusted meta-analysis. Obesity 2013.

38 Hawley NL, Johnson W, Hart CN, et al. Gestational weight gain among American Samoan women and its impact on delivery and infant outcomes. BMC Pregnancy Childbirth 2015;15:10.

39 Barker $\mathrm{H}$. Bravo for the Marshallese: regaining control in a postnuclear, post-colonial world. Cengage learning, 2012.

40 Zak D. A ground zero forgotten: the Marshall Islands, once a U.S. nuclear site, face oblivion again. Available: http://www. washingtonpost.com/sf/national/2015/11/27/a-ground-zeroforgotten/?utm_term=.d9dee192cdc5 [Accessed 3 Oct 2017].
41 Cortes LM, Gittelsohn J, Alfred J, et al. Formative research to inform intervention development for diabetes prevention in the Republic of the Marshall islands. Health Educ Behav 2001;28:696-715.

42 Ayers BL, Hawley NL, Purvis RS, et al. Providers' perspectives of barriers experienced in maternal health care among Marshallese women. Women Birth 2018:31:e294-301.

43 Ayers BL, Purvis RS, Bing WI, et al. Structural and Socio-cultural barriers to prenatal care in a US Marshallese community. Matern Child Health J 2018;22:1067-76.

44 Israel BA, Coombe CM, Cheezum RR, et al. Community-Based participatory research: a capacity-building approach for policy advocacy aimed at eliminating health disparities. Am J Public Health 2010;100:2094-102.

45 Ayers B, Bogulski C, Haggard-Duff L, et al. Healthy start program: a program to aid Marshallese mothers access to quality care in Arkansas, 2019.

46 Creswell JW. Research design: qualitative, quantitative, and mixed methods approaches. Sage Publications, 2009.

47 Bergman M. Advances in mixed methods research: theories and applications. Sage Publications Ltd, 2008.

48 Creswell J, Clark P V, Gutmann M, et al. Advanced mixed methods research designs. In: Tashakkori A, Teddlie C, eds. Handbook of mixed methods in social and behavioral research. Sage, 2003: 209-40.

49 Tang X, Andres A, West DS, et al. Eating behavior and weight gain during pregnancy. Eat Behav 2020;36:101364.

50 Harris PA, Taylor R, Thielke R, et al. Research electronic data capture (REDCap)--a metadata-driven methodology and workflow process for providing translational research informatics support. J Biomed Inform 2009;42:377-81.

51 Scott A, Shreve M, Ayers B, et al. Beliefs and experiences of Marshallese migrants: an exploratory study. Public Health Nutr 2016:1-10.

52 Charmaz K. Constructing Grounded theory: a practical guide through qualitative analysis. Sage Publications Ltd, 2006.

53 Ayers BL, Purvis RS, Bing W. Maternal health beliefs in a Pacific Islander community. J Fam Community Health 2018.

54 McElfish PA, Long CR, Kohler PO, et al. Comparative effectiveness and maintenance of diabetes self-management education interventions for Marshallese patients with type 2 diabetes: a randomized controlled trial. Diabetes Care 2019;42:849-58.

55 Charmaz K. 'Discovering' chronic illness: using grounded theory. Soc Sci Med 1990;30:1161-72.

56 Charmaz K. Teaching theory construction with initial Grounded theory tools: a reflection on lessons and learning. Qual Health Res 2015;25:1610-22.

57 Kaholokula JK, Wilson RE, Townsend CKM, et al. Translating the Diabetes Prevention Program in Native Hawaiian and Pacific Islander communities: the PILI 'Ohana Project. Trans/ Behav Med 2014;4:149-59.

58 Holzer JK, Ellis L, Merritt MW. Why we need community engagement in medical research. J Investig Med 2014;62:851-5.

59 De las Nueces D, Hacker K, DiGirolamo A, et al. A systematic review of community-based participatory research to enhance clinical trials in racial and ethnic minority groups. Health Serv Res 2012;47:1363-86.

60 Minkler M. Ethical challenges for the "outside" researcher in community-based participatory research. Health Educ Behav 2004;31:684-97.

61 Vaughn LM, Jacquez F, Lindquist-Grantz $R$, et al. Immigrants as research partners: a review of immigrants in community-based participatory research (CBPR).. J Immigr Minor Health 2016.

62 Carpenter D, Nieva V, Albaghal T, et al. Development of a planning tool to guide research dissemination. In: Henriksen K, Battles JB, Marks ES, et al, eds. Advances in patient safety: from research to implementation. Volume 4: Programs, Tools, and Products, chap 8. Agency for Healthcare Research and Quality, 2005: 83-91. 